\title{
SMOOTH EXTENSIONS IN INFINITE DIMENSIONAL BANACH SPACES
}

BY

\author{
PETER RENZ( $\left.{ }^{1}\right)$
}

\begin{abstract}
If $B$ is $l_{p}(\omega)$ or $c_{0}(\omega)$ we show $B$ has the following extension property. Any homeomorphism from a compact subset $M$ of $B$ into $B$ may be extended to a homeomorphism of $B$ onto $B$ which is a $C^{\infty}$ diffeomorphism on $B \backslash M$ to its image in $B$. This is done by writing $B$ as a direct sum of closed subspaces $B_{1}$ and $B_{2}$ both isomorphically isometric to $B$ so that the natural projection of $K$ into $B_{1}$ along $B_{2}$ is one-to-one (see H. H. Corson, contribution in Symposium on infinite dimensional topology, Ann. of Math. Studies (to appear)). With $K, B, B_{1}$ and $B_{2}$ as above a homeomorphism of $B$ onto itself is constructed which leaves the $B_{1}$-coordinates of points in $B$ unchanged, carries $K$ into $B_{1}$ and is a $C^{\infty}$ diffeomorphic map on $B \backslash K$. From these results the extension theorem may be proved by standard methods.
\end{abstract}

1. Introduction, outline and preliminaries. Our main result is the following extension theorem.

THEOREM 4.1. Let $B$ be $C_{0}(\omega)$ or $l_{p}(\omega)$ and let $M$ and $N$ be compact subsets of $B$. Let $f$ be a homeomorphism mapping $M$ onto $N$. There is an extension $f^{*}$ of $f$ to a homeomorphism of $B$ onto itself such that $f^{*}$ restricted to $B \backslash M$ is a $C^{\infty}$ diffeomorphism of $B \backslash M$ onto $B \backslash N$.

We start with the following Splitting Theorem which is an immediate consequence of the results and techniques of Corson [6].

Splitting Theorem (Corson). Let $B$ be $C_{0}(\omega)$ or $l_{p}(\omega)$. Let $K$ be a $\sigma$-compact subset of $B$. Then $B$ may be written as an internal direct sum $B=B_{1}+B_{2}$ where $B_{1}$ and $B_{2}$ are both isomorphic to $B$ and where the projection $\pi_{1}$ of $B$ into $B_{1}$ along $B_{2}$ yields a one-to-one map when restricted to $K$.

This theorem will be applied to a compact set $K$ and $\pi_{1}(K)$ will be denoted $K_{1}$. In this case $\pi_{1}$ yields a homeomorphism of $K$ onto $K_{1}$. Further, there is a continuous mapping $g$ of $K_{1}$ into $B_{1}$ such that $K=\left\{k_{1}+g\left(k_{1}\right) \mid k_{1} \in K_{1}\right\}$. By a slight abuse of the language we call $\left\{k_{1}+g\left(k_{1}\right) \mid k_{1} \in K_{1}\right\}$ the graph of $g$.

Thus we see that the compact set $K$ is the graph of a continuous function $g$ from some set $K_{1}$ which lies in a subspace $B_{1}$ of infinite deficiency in $B$ into $B_{2}$ a complement of $B_{1}$ in $B$.

Presented to the Society, April 26, 1969 under the title Smooth extensions and smooth extractions; received by the editors January 7, 1971 and, in revised form, April 26, 1971.

AMS 1970 subject classifications. Primary 57D50, 54C20; Secondary 58B10.

Key words and phrases. Extension of homeomorphisms, infinite dimensional Banach spaces, $C^{\infty}$ extensions, compact subsets of infinite dimensional spaces.

( ${ }^{1}$ The preparation of this paper was aided by support from Reed College.

Copyright (C) 1972, American Mathematical Society 
The above observation about compact sets in certain infinite dimensional Banach spaces leads to a proof of the extension theorem by fairly standard methods once we have established the following somewhat technical result on smoothly flattening graphs into subspaces.

THEOREM 3.1. Let $B$ be the direct internal sum of Banach spaces $B_{1}$ and $B_{2}$. Let each of $B_{1}$ and $B_{2}$ satisfy at least one of the conditions of being separable or admitting $C^{\infty}$ partitions of unity subordinate to any open cover. Let $K_{1}$ be a compact subset of $B_{1}$ and let $K=\left\{k_{1}+g\left(k_{1}\right) \mid k_{1} \in K_{1}\right\}$ where $g$ is a continuous map of $K_{1}$ into $B_{2}$. Then there is a homeomorphism $d$ of $B$ onto $B$ such that

(i) $d(k)=\pi_{1}(k)$ for all $k \in K$, where $\pi_{1}$ is the natural projection of $B$ into $B_{1}$ along $B_{2}$,

(ii) $\pi_{1} \circ d=\pi_{1}$,

(iii) $d$ restricted to $B \backslash K$ is a $C^{p}$ diffeomorphism of $B \backslash K$ onto $B \backslash K_{1}$.

If both spaces allow $C^{\infty}$ norms or are separable, then $p$ in (iii) may be taken to be $\infty$.

If one merely wants $d$ to be a homeomorphism, $d$ may be defined by $d\left(x_{1}+x_{2}\right)$ $=x_{1}+x_{2}-g^{*}\left(x_{1}\right)$, where $g^{*}$ is any continuous extension of $g$ to a map from $X_{1}$ to $X_{2}$. Such an extension of $g$ is easily constructed. This shows that the topological version of Theorem 3.1 follows immediately from the Splitting Theorem. The difficulties which must be overcome here arise from the requirement of smoothness.

The reader will notice that the constructions presented here may be used in spaces other than $c_{0}(\omega)$ and $p(\omega)$. For separable spaces one need only check that the spaces admit an appropriate splitting (see Corson [6]). For nonseparable spaces one also needs smooth partitions of unity to carry through the construction of $\S 3$. For the constructions of the other sections it is sufficient that the space admit some suitable smooth function (for instance, a continuous norm which is $C^{p}$ except at the origin). The theory of the existence of smooth partitions of unity (Bonic and Frampton [5]) applies to separable spaces. However, we have stated our results so as to take advantage of any extension of this theory to nonseparable spaces.

The splitting and flattening theorems above were devised to take compact subsets of infinite dimensional Banach spaces into subspaces of infinite deficiency. Once a compact set has been carried into a subspace of infinite deficiency it may be extracted by a refinement of the process used by Bessaga [3]. In this way with some effort and delicacy one may prove that the removal of closed locally compact subsets from a smooth infinite dimensional $B$-manifold $M$ leaves the differentiable structure of $M$ unchanged for many Banach spaces $B$ (for details, see the author's dissertation [20]).

Several authors have considered or used results on the negligibility of various spacial sorts of subsets of various infinite dimensional manifolds. For the topological case, see Bessaga's notes [4] which survey the field. This author does not know of such a survey in the differentiable case. However, we have the work of Bessaga [3], Eells and Kuiper [11], Martens [16], and Cutler [7] to mention a few. 
Results of this sort must be compared with those obtained by a very different and much deeper approach. A series of results starting with Kuiper's paper showing that the general linear group of $l_{2}(\omega)$ is contractible [13] has led to the characterization up to diffeomorphism of most common infinite dimensional Banach manifolds $M$ by the homotopy type of $M$. (See papers of Kuiper and Burghelea [14], Moulis [17], Eells and Elworthy [10] for results on manifolds. See papers of Neubauer [18], Edelstein, Mitjagin and Semenov [9] for papers on contractibility of general linear groups of Banach spaces.) This deep theory together with results known in the topological case leads to the fact that removal of closed $\sigma$-compact subsets does not change the $C^{\infty}$ structure of such manifolds.

The approach to negligibility presented here has the advantage of being more elementary. It may be extended to nonseparable spaces in so far as these spaces admit suitable splittings (one only needs $B_{1}$ to be of infinite deficiency in $B$ ) and smooth partitions of unity. The needed splitting theorem is not hard to prove for nonseparable spaces. However, the question of partitions of unity in such spaces is still open even when the spaces have smooth norms. The techniques used here do not lead to the stronger classification of manifolds mentioned above.

The following outlines the steps in establishing the extension theorem. In $\S 1$ we introduce the notion of smooth approximation and prove a series of lemmas leading to our main lemma on flattening graphs into subspaces (Lemma 2.4). In $\S 3$ we show that continuous functions on compact sets in the context of Theorem 3.1 always satisfy the smooth approximability condition of Lemma 2.4. This leads immediately to a proof of Theorem 3.1. In $\S 4$ we use the Splitting Theorem of Corson and the remarks about compact sets and graphs of this introductory section to show that Theorem 3.1 may be applied in a symmetrical way to obtain a special result on flattening compact sets in $c_{0}(\omega)$ or $l_{p}(\omega)$. From this result the proof of Theorem 4.1 is direct.

The author expresses his debt to Harry Corson who suggested the problem which led to these results. Professor Corson made many helpful strategic and tactical suggestions during this work. In particular, he pointed out that it was possible to obtain extension results from flattening results by the methods used here. The outline of this extension process in the topological case comes from Klee [12]. The author's thesis and its results given here depend upon the general methods and to some extent upon specific results of Klee's work on infinite dimensional topology. It is my pleasure to acknowledge this debt to Professor Klee and his work.

Almost all of the notation used is standard. The symbol $\boldsymbol{R}$ stands for the real numbers and when $\boldsymbol{N}$ appears for an index set it denotes the positive integers. If a function is $p$ times continuously differentiable we say it is $C^{p}$. If a function is $C^{p}$ for all $p$ in $N$ we say it is $C^{\infty}$. We will use $D f, f^{\prime}$, or $(d / d x) f$ to denote the derivative of a function $f$, the notation chosen will be appropriate to the context. The abbreviations cl and int will be used for the topological operations of closure and interior. 
2. Smooth approximation and the flattening of graphs. Let $X$ and $Y$ be normed linear spaces and let $U$ be an open subset of $X$. We say a sequence of functions $\left\{f_{n} \mid n \in N\right\}$ from $X$ into $Y$ smoothly approximates the function $f$ from $X$ to $Y$ on $U$ provided

(i) $\left\|f_{n}(x)-f(x)\right\|$ tends to zero uniformly in $x$ as $n$ tends to infinity, and

(ii) $f_{n}$ is eventually constant as a function of $n$ locally on $U$ (i.e. for each $u \in U$ there is a neighborhood $U(u)$ of $u$ and an integer $n(u)$ such that $f_{k}(x)=f_{m}(x)$ for all $x \in U(u)$ and all integers $k, m \geqq n(u))$.

LEMMA 2.1. Let $X$ and $Y$ be normed linear spaces and let the map fof $X$ into $Y$ be smoothly approximable on $U$ by a sequence of $C^{p}$ functions. There is a function $F$ from $\boldsymbol{R} \times X$ into $Y$ such that

(i) $F(r, \cdot)$ is eventually constant as a function of $r$ as $r$ tends to 1 locally on $U$.

(ii) $F$ is continuous and $F(r, x)$ is $C^{\infty}$ in $r$ and $C^{p}$ in $x$ if $r<1$ or $x \in U$.

(iii) $F(r, \cdot)=f(\cdot)$ if $r \geqq 1$.

(iv) $\left\|D_{1} F(r, x)\right\| \leqq \frac{1}{2}$ for all $r$ in $R$ and $x$ in $X$.

Proof. Let $h_{n}$ be a sequence of nondecreasing infinitely differentiable functions from $\boldsymbol{R}$ to $\boldsymbol{R}$ satisfying

$$
\begin{array}{ll}
h_{n}(x)=0 & \text { if } x \leqq 1-2^{1-n}, \\
h_{n}(x)=1 & \text { if } x \geqq 1-2^{-n} \text { for all } n \text { in } N .
\end{array}
$$

Let $a_{n}$ be a sequence of real numbers such that $h_{n}^{\prime}(x) \leqq a_{n}$ for all $x$ in $\boldsymbol{R}$ and all $n$ in $N$. By our hypothesis we may find a sequence $f_{n}$ of $C^{p}$ functions smoothly approximating $f$ on $U$ satisfying $\left\|f_{n}-f\right\| \leqq \frac{1}{2} a_{k}^{-2}$ for all $k \leqq n+1$ for all $n$ in $N$. We define $F$ by

$$
F=f_{1}+\sum h_{n+1} \cdot\left(f_{n+1}-f_{n}\right) \quad(n \in N) .
$$

Since the series defining $F$ has only finitely many nonzero terms in some neighborhood $\boldsymbol{R} \times U(x)$ of a point $(r, x)$ with $x$ in $U$ and since each $h_{k}(r)$ eventually becomes identically 1 as $r$ tends to 1 property (i) is true. Similarly if $r<1$, the series defining $F$ consists of only finitely many terms on the neighborhood $\left(-\infty, \frac{1}{2}(r+1)\right)$ $\times X$ of $(r, x)$. Since each term of the series is $C^{\infty}$ in $r$ and $C^{p}$ in $x$, it follows that the function $F$ which is a finite sum in a neighborhood of any point $(r, x)$ such that $r<1$ or $x \in U$ is $C^{\infty}$ in $r$ and $C^{p}$ in $x$ on $\{(r, x) \in(\boldsymbol{R} \times X) \mid r<1$ or $x \in U\}$ as required in (ii).

The mean value theorem ensures that $a_{k} \geqq 2^{k}$. The following estimate shows that the series defining $F$ is absolutely and uniformly convergent on $\boldsymbol{R} \times X$.

$$
\begin{aligned}
\sum \| h_{k+1}(r)\left(f_{k+1}(x)\right. & \left.-f_{k}(x)\right) \| & & \\
& \leqq \sum\left|h_{k+1}(r)\right|\left\|f_{k+1}(x)-f_{k}(x)\right\| & & (k \in N) \\
& \leqq \sum 1\left(\left\|f_{k+1}(x)-f(x)\right\|+\left\|f(x)-f_{k}(x)\right\|\right) & & (k \in N) \\
& \leqq \sum\left(\frac{1}{2} a_{k+1}^{-2}+\frac{1}{2} a_{k}^{-2}\right) & & (k \in N) \\
& <\infty & & (k \in N) .
\end{aligned}
$$


Hence $F$ is the sum of an absolutely and uniformly convergent series of continuous functions and therefore $F$ is a continuous function on $\boldsymbol{R} \times X$. Thus (ii) is established. If $r \geqq 1$, then $h_{k+1}(r)=1$ for all $k$ in $N$ and the series defining $F(r, \cdot)$ is a telescoping series with limit $f(\cdot)$. Thus (iii) is established.

The following estimate shows that the derived series is absolutely and uniformly convergent; hence, by a standard theorem (Dieudonné $[8,8.6 .5]$ ) term by term differentiation is justified and $\left\|D_{1} F\left(r, x_{1}\right)\right\| \leqq \frac{1}{2}$. We have

$$
\begin{aligned}
\sum \| h_{n+1}^{\prime}(r)\left(f_{n+1}\left(x_{1}\right)-f_{n}(\right. & \left.\left.x_{1}\right)\right) \| & & \\
& \leqq \sum\left|h_{n+1}^{\prime}(r)\right|\left(\left\|f_{n+1}-f\right\|+\left\|f-f_{n}\right\|\right) & & (n \in N) \\
& \leqq \sum a_{n+1}\left(\frac{1}{2} a_{n+1}^{-2}+\frac{1}{2} a_{n+1}^{-2}\right) & & (n \in N) \\
& \leqq \sum a_{n+1}^{-1} & & (n \in N) \\
& \leqq \frac{1}{2} & & (n \in N),
\end{aligned}
$$

since $a_{n} \geqq 2^{n}$ for $n$ in $\boldsymbol{N}$. This establishes (iv).

The context and notation of the next lemma are as follows. The normed linear space $X$ is the direct internal sum of subspaces $X_{1}$ and $X_{2}$. We assume the natural projections of $X$ into $X_{1}$ and $X_{2}$ have operator norm less than or equal to 1 . There is a continuous function $f$ mapping $X_{1}$ to $X_{2}$ and $G f=\left\{x_{1}+f\left(x_{1}\right) \mid x_{1} \in X_{1}\right\}$ is called the graph of $f$. The set $G f$ is a closed subset of $X=X_{1}+X_{2}$ since $f$ is continuous.

Lemma 2.2. Let $X, X_{1}$ and $X_{2}$ be as above. Let $f: X_{1} \rightarrow X_{2}$ be the uniform limit of $C^{p}$ functions. Assume the norm of $X_{2}$ is $C^{p}$ on $X_{2} \mid\{0\}$. (Alternatively, assume $X_{2}$ is separable.) There is a continuous function $\phi$ from $X$ to $[0,1]$ which is $C^{p}$ (alternatively, $\left.C^{\infty}\right)$ on $Y=X \backslash G f$ such that $\left\|D_{2} \phi\right\| \leqq \frac{1}{2}$ and $\phi(x)=1$ exactly when $x \in G f$.

Proof. Let $a, b, c$ and $d$ be positive numbers with $a<b$. Let $h$ be a nonincreasing $C^{\infty}$ function from $\boldsymbol{R}$ to $\boldsymbol{R}$ satisfying

$$
\begin{aligned}
c & \geqq h(r)=h(0)>0 & & \text { whenever } r \leqq a, \\
h(r) & =0 & & \text { whenever } r \geqq b, \\
\left|h^{\prime}(r)\right| & \leqq d & & \text { for all } r \text { in } R .
\end{aligned}
$$

Let $\varepsilon$ be a positive number and let $g$ be a $C^{p}$ function from $X_{1}$ to $X_{2}$ such that $\left\|g\left(x_{1}\right)-f\left(x_{1}\right)\right\|<\varepsilon$. Then $\psi\left(x_{1}+x_{2}\right)=h\left(\left\|x_{2}-g\left(x_{1}\right)\right\|\right)$ is a nonnegative $C^{p}$ function on $X$ satisfying

$$
\begin{aligned}
c & \geqq \psi\left(x_{1}+x_{2}\right)=h(0)>0 & & \text { if }\left\|x_{2}-f\left(x_{1}\right)\right\| \leqq a-\varepsilon, \\
\psi\left(x_{1}+x_{2}\right) & =0 & & \text { if }\left\|x_{2}-f\left(x_{1}\right)\right\| \geqq b+\varepsilon, \\
\left\|D_{2} \psi\left(x_{1}+x_{2}\right)\right\| & \leqq d & & \text { for all }\left(x_{1}+x_{2}\right) \in \dot{X} .
\end{aligned}
$$

The nonnegativity and first two properties of $\psi$ are evident. Off of

$$
G g=\left\{x_{1}+g\left(x_{1}\right) \mid x_{1} \in X_{1}\right\}
$$


the function $\psi$ is a composition of $C^{p}$ functions, while at each point of $G g$ the function $\psi$ is locally constant. Thus $\psi$ is at least $C^{p}$ on $X$. The bound on the norm of $D_{2} \psi$ is established by taking $D_{2}$ by the usual methods (Lang [15]) and using the fact that the operator norm of the derivative of the norm of any Banach space is less than or equal to 1 .

We fix sequences $a_{n}, b_{n}, c_{n}$ and $\varepsilon_{n}$ of positive numbers such that

$a_{n}<b_{n}$ and $b_{n+1}+\varepsilon_{n+1}<a_{n}-\varepsilon_{n}$ for all $n$ in $N$,

$b_{n}$ tends to zero as $n$ tends to infinity,

$1 \geqq \sum c_{n}(n \in N)$, and

$\frac{1}{2} \geqq \sum d_{n}(n \in N)$,

(e.g., $a_{n}=2^{-2 n}, b_{n}=2 a_{n}, c_{n}=2^{-4 n}, d_{n}=\frac{1}{2} c_{n}$, and $\varepsilon_{n}=2^{-4(n+1)}$ ).

We take a sequence $\psi_{n}$ of nonnegative $C^{p}$ functions on $X$ satisfying conditions (*) with $a=a_{n}, b=b_{n}, c=c_{n}, d=d_{n}$ and $\varepsilon=\varepsilon_{n}$. We set

$$
\psi^{*}(x)=\sum \psi_{n}(x) \quad(n \in N) .
$$

The conditions ensure that $\psi^{*}$ is an absolutely and uniformly convergent sum of continuous functions and hence continuous.

Since $f$ is the uniform limit of $C^{p}$ functions, $f$ is continuous. If $\left(x_{1}+x_{2}\right) \in Y$, then $x_{2} \neq f\left(x_{1}\right)$ and for some $n\left\|x_{2}-f\left(x_{1}\right)\right\|>b_{n}+\varepsilon_{n}$. Since this inequality holds at $x_{1}+x_{2}$ and since the functions involved are continuous, it holds in a neighborhood of $x_{1}+x_{2}$. But this inequality implies $\psi_{k}$ vanishes for $k \geqq n$. Hence $\psi^{*}$ is locally a finite sum of $C^{p}$ functions and thus $C^{p}$ on $Y$.

Each point $x_{1}+x_{2}$ of $G f$ satisfies $0=\left\|x_{2}-f\left(x_{1}\right)\right\|<a_{n}-\varepsilon_{n}$ for all $n$ in $N$. Thus $\psi^{*}$ equals the constant

$$
d^{*}=\sum \psi_{n}\left(x_{1}+f\left(x_{1}\right)\right) \quad(n \in N)
$$

on $G f$. Clearly, $d^{*}>\psi^{*}\left(x_{1}+x_{2}\right)$ if $\left(x_{1}+x_{2}\right) \in Y$, since $\psi_{n}\left(x_{1}+f\left(x_{1}\right)\right)$ is positive and $\psi_{n}\left(x_{1}+x_{2}\right) \leqq \psi_{n}\left(x_{1}+f\left(x_{1}\right)\right)$ for all $n$ in $N$ while only finitely many of the terms $\psi_{n}\left(x_{1}+x_{2}\right)$ are nonzero. Thus $\psi^{*}$ attains its supremum $d^{*}$ precisely on the set $G f$.

The derived series for $D_{2} \psi^{*}$ is absolutely and uniformly convergent in view of the bounds on $\left\|D_{2} \psi_{n}\right\|$. Thus, term by term differentiation is justified and $\left\|D_{2} \psi^{*}\right\|$ $\leqq \frac{1}{2}$. The function $\psi^{*}$ has most of the properties we require. To obtain a function having all the desired properties, it is sufficient to set $\phi=\psi^{*}+\left(1-d^{*}\right)$. This insures that the supremum of $\phi$ which is obtained only on $G f$ is equal to 1 . It is clear that $\phi$ is as differentiable as $\psi^{*}$ and that $\left\|D_{2} \phi\right\|=\left\|D_{2} \psi^{*}\right\| \leqq \frac{1}{2}$. Thus $\phi$ has all the properties required in the lemma.

In this construction we may use any $C^{p}$ function $N(\cdot)$ on $X_{2}$ in place of the norm $\|\cdot\|$ of $X_{2}$ provided $N$ is a norm and $N(x) \leqq\|x\|$ for all $x$ in $X_{2}$. To establish the alternative reading it suffices to show that any separable Banach space $Y$ admits a possibly weaker norm $N(\cdot)$ with the above properties. To do this let $Y$ be a separable Banach space and let $\left\{y_{n} \mid n \in N\right\}$ be a sequence of points of norm 1 in $Y$ whose range is dense in the unit sphere of $Y$. We may construct a sequence of 
linear functionals $\left\{f_{n} \mid n \in N\right\}$ on $Y$ each of norm 1 such that $f_{n}\left(y_{n}\right)=1$ for $n \in N$. It is easily seen that

$$
N(y)=\left(\sum_{n=1}^{\infty} 2^{-n}\left(f_{n}(y)\right)^{2}\right)^{1 / 2}
$$

is a $C^{\infty}$ norm on $Y$ with the desired properties. This shows that every separable space admits a smaller and possibly weaker $C^{\infty}$ norm and thus establishes the alternative reading of the lemma. An alternative approach is to use the fact that any separable Banach space is isometrically isomorphic with a closed subspace of $C[0,1]$ which admits a $C^{\infty}$ norm of the desired sort, namely the $\mathscr{L}_{2}$ norm.

We need the following version of Banach's contraction principle. We omit the proof which is simply an exercise in the properties of contraction maps.

Lemma 2.3. Let $X_{1}$ be a normed linear space and $X_{2}$ be a Banach space. Let $X=X_{1}+X_{2}$ and let $h$ be a continuous function from $X$ to $X_{2}$ such that

$$
\left\|h\left(x_{1}+x_{2}\right)-h\left(x_{1}+x_{2}^{\prime}\right)\right\| \leqq \frac{1}{2}\left\|x_{2}-x_{2}^{\prime}\right\|
$$

for all $x_{1} \in X_{1}$ and $x_{2}, x_{2}^{\prime} \in X_{2}$. Let $d$ be a mapping defined by $d\left(x_{1}+x_{2}\right)=x_{1}+x_{2}$ $+h\left(x_{1}+x_{2}\right)$. Then $d$ is a homeomorphism of $X$ onto itself, and $d$ is a $C^{p}$ diffeomorphism when restricted to any open set on which $h$ is $C^{p}$.

These lemmas lead to a proof of our main lemma.

Lemma 2.4. Let $X_{1}$ be a normed linear space and $X_{2}$ a Banach space with $C^{p}$ norm. (Alternatively, let $X_{2}$ be a separable Banach space.) Let $f: X_{1} \rightarrow X_{2}$ be smoothly approximable by $C^{p}$ functions on $U$. Let Gf be the graph off. Let $K_{1}=X_{1} \backslash U$ and let $K=\left\{k_{1}+g\left(k_{1}\right) \mid k_{1} \in K_{1}\right\}$. Then there is a homeomorphism $d$ of $X_{1}+X_{2}=X$ onto itself such that

(i) $d(G f)=\pi_{1}(G f)$,

(ii) $d \circ \pi_{1}=\pi_{1}$,

(iii) $d$ restricted to $X \backslash K$ is a $C^{p}$ diffeomorphism of $X \backslash K$ onto $X \backslash K_{1}$. (Alternatively,

(iii) d restricted to $X \backslash K$ is a $C^{\infty}$ diffeomorphism of $X \backslash K$ onto $X \backslash K_{1}$.)

Proof. By the conditions of the theorem there exist functions $F$ and $\phi$ satisfying the conditions in Lemmas 2.1 and 2.2 with respect to $X_{1}, X_{2}$, and $f$. We define $h\left(x_{1}+x_{2}\right)$ for $\left(x_{1}+x_{2}\right) \in X$ by

$$
h\left(x_{1}+x_{2}\right)=F\left(\phi\left(x_{1}+x_{2}\right), x_{1}\right) .
$$

The function $h$ defined above is the composition of continuous functions and thus continuous. If $\left(x_{1}+x_{2}\right) \notin G f$ then $\phi$ is $C^{p}$ at $x_{1}+x_{2}$ by the conditions of Lemma 2.2, and $F$ is $C^{p}$ at $\left(\phi\left(x_{1}+x_{2}\right), x_{1}\right)$ because $\phi\left(x_{1}+x_{2}\right)<1$. Thus, $h$ is $C^{p}$ on $X \backslash G f$. If $\left(a_{1}+a_{2}\right) \in(G f \backslash K)$, then $a_{1} \in U$. Thus, as in Lemma 2.1, there is a neighborhood $U\left(a_{1}\right)$ of $a_{1}$ and an $r\left(a_{1}\right)<1$ such that $F\left(r, x_{1}\right)=f\left(x_{1}\right)$ if $r>r\left(a_{1}\right)$ and $x_{1} \in U\left(a_{1}\right)$. 
Now $\left(a_{1}+a_{2}\right) \in G f$ so $\phi\left(a_{1}+a_{2}\right)=1$. Since $\phi$ is continuous, there is a neighborhood $V$ of $a_{1}+a_{2}$ such that $\phi\left(x_{1}+x_{2}\right)>r\left(a_{1}\right)$ and $x_{1} \in U\left(a_{1}\right)$ for all $\left(x_{1}+x_{2}\right) \in V$. For $\left(x_{1}+x_{2}\right) \in V$,

$$
F\left(\phi\left(x_{1}+x_{2}\right), x_{1}\right)=F\left(\frac{1}{2}\left(r\left(a_{1}\right)+1\right), x_{1}\right)=f\left(x_{1}\right) .
$$

Thus $h$ is $C^{p}$ on $V$ since $\frac{1}{2}\left(r\left(a_{1}\right)+1\right)$ is a $C^{\infty}$ function less than 1 on $V$ and $F\left(r, x_{1}\right)$ is a $C^{p}$ function of $x_{1}$ if $r<1$. This shows $h$ is a $C^{p}$ function on $(X \backslash G f) \cup(G f \backslash K)$ $=X \backslash K$. It also shows that if $\left(x_{1}+x_{2}\right) \in(G f \backslash K)$ and $V$ is as above, then $D_{2} h=0$ on $V$ since $h$ does not depend on the second coordinate within $V$.

Computing $D_{2} h$ we have

$$
D_{2} h\left(x_{1}+x_{2}\right)=D_{1} F\left(\phi\left(x_{1}+x_{2}\right), x_{1}\right) \circ D_{2} \phi\left(x_{1}+x_{2}\right) .
$$

The bounds on the norms of $D_{1} F$ and $D_{2} \phi$ give us $\left\|D_{2} h\left(x_{1}+x_{2}\right)\right\| \leqq \frac{1}{2} \cdot \frac{1}{2}=\frac{1}{4}$ when $\left(x_{1}+x_{2}\right) \notin G f$. Since $h$ is continuous and $X \backslash G f$ is dense in $X$, we may use the mean value theorem (Corollary 1, Proposition 17, Chapter 1, $\S 4$ of Lang [15]) to show that the inequality

$$
\left\|h\left(x_{1}+x_{2}\right)-h\left(x_{1}+x_{2}^{\prime}\right)\right\| \leqq \frac{1}{4}\left\|x_{2}-x_{2}^{\prime}\right\|
$$

holds for all $x_{2}, x_{2}^{\prime} \in X_{2}$ for all $x_{1} \in X_{1}$. Thus Lemma 2.3 applies to the function $d\left(x_{1}+x_{2}\right)=x_{1}+x_{2}-h\left(x_{1}+x_{2}\right)$ defined for all $\left(x_{1}+x_{2}\right) \in X$. It is clear from the definition of $d$ that (ii) holds.

Applying Lemma 2.3 to the function $d$ we see that $d$ is a homeomorphism of $X$ onto itself. Since $d$ is $C^{p}$ on $X \backslash K$ it is a $C^{p}$ diffeomorphism of $X \backslash K$ onto $d\left(X \backslash K_{1}\right)$.

If $\left(x_{1}+x_{2}\right) \in G f$, then $h\left(x_{1}+x_{2}\right)=F\left(1, x_{1}\right)=f\left(x_{1}\right)=x_{2}$ and

$$
d\left(x_{1}+x_{2}\right)=x_{1}+x_{2}-h\left(x_{1}+x_{2}\right)=x_{1}+x_{2}-x_{2}=x_{1} .
$$

This establishes (i). In view of the above paragraph, to complete the proof it is sufficient to show that $d(X \backslash K)=X \backslash K_{1}$, or equivalently $d(K)=K_{1}$. But this follows from (i) and the definitions of $K$ and $K_{1}$.

Lemma 2.4 outlines conditions under which certain kinds of subsets $K$ of $X=X_{1}+X_{2}$ may be carried smoothly into $X_{1}$. We will see that the conditions of this lemma are easily satisfied when the subset $K$ is compact and $X$ is an infinite dimensional Banach space. There are two requirements for applying Lemma 2.4. First, the set $K$ must be the graph of a continuous function from $X_{1}$ to $X_{2}$ and second, that function must be smoothly approximable on $U=X_{1} \mid \pi_{1}(K)$. The Splitting Theorem shows circumstances under which compact sets may be regarded as graphs of continuous functions. We now take up the problem of smooth approximation.

3. Smooth approximation of functions. Let $B$ be a Banach space which is the internal direct sum of subspaces $B_{1}$ and $B_{2}$. Let $K_{1}$ be a subset of $B_{1}$ and let $g$ be a continuous function from $K_{1}$ to $B_{2}$. Let $K=\left\{k_{1}+g\left(k_{1}\right) \mid k_{1} \in K_{1}\right\}$. When may $g$ be extended to a function $f$ mapping $B_{1}$ into $B_{2}$ so that $F, B_{1}, B_{2}$, and $K_{1}$ satisfy the hypotheses of Lemma 2.4 ? 
We show that it is sufficient to assume $K$ (and hence $K_{1}$ ) is compact and that each of $B_{1}$ and $B_{2}$ is either separable or admits $C^{p}$ partitions of unity subordinate to any open cover. On $B_{2}$ either of these assumptions is sufficient to apply Lemma 2.4. We show below that either of these assumptions on $B_{1}$ will insure that $g$ has an extension $f$ satisfying the conditions above.

Let $B_{1}$ admit $C^{p}$ partitions of unity. We will construct a series of $C^{p}$ functions $f_{n}$ whose partial sums approximate $g$ on $K_{1}$ and smoothly approximate $f=\sum_{n=1}^{\infty} f_{n}$ on $B_{1} \backslash K_{1}$. Thus $f$ is an extension of $g$ of the desired sort.

Since $K_{1}$ is compact and $g$ is continuous, $g$ is uniformly continuous on $K_{1}$. Let $\mathscr{V}_{1}$ be a finite cover of $K_{1}$ by open sets in $B_{1}$ such that $\left\|f(k)-f\left(k^{\prime}\right)\right\| \leqq 1$ for all $k$, $k^{\prime} \in V \cap K$ for all $V \in \mathscr{V}_{1}$. We further assume that the diameter of each $V \in \mathscr{V}_{1}$ is less than or equal to 1 . For each $V \in \mathscr{V}_{1}$ select a $k_{1}(V) \in V$. Let $\left\{\phi_{V} \mid V \in \mathscr{V}_{1}\right\}$ $\cup\left\{\phi_{U}\right\}$ be a $C^{p}$ partition of unity subordinate to the cover $\mathscr{V}_{1} \cup\{U\}$ of $B_{1}$. Define $f_{1}$ by

$$
f_{1}=\sum g\left(k_{1}(V)\right) \phi_{V} \quad\left(V \in \mathscr{V}_{1}\right)
$$

Then

$$
\begin{aligned}
\left\|g(k)-f_{1}(k)\right\| & =\left\|\sum_{V \in \mathscr{V}_{1}} g\left(k_{1}(V)\right) \phi_{V}(k)-\sum_{V \in \mathscr{V}_{1}} f(k) \phi_{V}(k)\right\| \\
& \leqq \sum_{V \in \mathscr{V}_{1}}\left\|g\left(k_{1}(V)\right)-f(k)\right\||\phi(k)| \\
& \leqq \sum_{V \in \mathscr{V}_{1}} 1 \cdot \phi_{V}(k) \leqq 1=2^{0}
\end{aligned}
$$

for every $k \in K$. This defines a $C^{p}$ function $f_{1}$ which approximates $g$ on $K$ to within 1 and vanishes off of the neighborhood $\bigcup \mathscr{V}_{1}$ of $K$. By the same process, we may produce a $C^{p}$ function $f_{2}$ which approximates $g-f_{1}$ on $K$ to within $\frac{1}{2}$ on $K$ and which vanishes off of $\cup \mathscr{V}_{2}$, where $\mathscr{V}_{2}$ is an open cover of $K$ in $B_{1}$ none of whose sets have diameter greater than $\frac{1}{2}$. In the construction we will have

$$
\begin{aligned}
\left\|f_{2}(x)\right\| & =\left\|\sum_{V \in \mathscr{V}_{2}}\left(g\left(k_{2}(V)\right)-f_{1}\left(k_{2}(V)\right)\right) \phi_{V}(x)\right\| \\
& \leqq \sum_{V \in \mathscr{V}_{2}}\left\|g\left(k_{2}(V)\right)-f_{1}\left(k_{2}(V)\right)\right\| \phi_{V}(x) \leqq \sum_{V \in \mathscr{V}_{2}} 1 \phi_{V}(x) \leqq 1 .
\end{aligned}
$$

Continuing in this way the sequence $f_{n}$ is constructed so that

(i) $\left\|g(k)-\sum_{k=1}^{n} f_{n}(k)\right\| \leqq 2^{1-n}$ for all $k \in K$ and $n=1,2,3, \ldots$,

(ii) $\left\|f_{n}\left(b_{1}\right)\right\| \leqq 2^{2-n}$ for $b_{1} \in B_{1}$ and $n=2,3,4, \ldots$,

(iii) $f_{n}$ vanishes off of the set $V_{n}=\bigcup \mathscr{V}_{n}$ and $\bigcap_{n=1}^{\infty} V_{n}=K$.

The final condition may be assured by insisting that the diameters of the $V_{n} \in \mathscr{V}_{n}$ be less than $2^{1-n}$. These conditions clearly ensure that $\sum f_{n}(n \in N)$ is uniformly convergent on $B_{1}$ and that $\sum_{i=1}^{n} f_{i}$ approximates $\sum_{i=1}^{\infty} f_{i}$ smoothly by $C^{p}$ functions on $B_{1} \backslash K$. Furthermore, it is clear that $g(k)=f(k)=\sum_{i=1}^{\infty} f(k)$ for all $k \in K$. This completes the case where $B_{1}$ admits $C^{p}$ partitions of unity. 
In the case where $B_{1}$ is separable we proceed similarly using weak open sets in place of strong open sets. This technique is suggested by the methods of Anderson [1]. Since $K$ is compact the weak and strong topologies on $K$ agree. Since $B_{1} \backslash K$ is Lindelöf in the strong topology and the identity map from the strong to weak topology is continuous, $U$ is Lindelöf in the weak topology. Since the weak topology is regular, $K$ is a weak $G_{\delta}$. In fact, we see that there is a family of weak open sets $\left\{U_{n} \mid n \in N\right\}$ such that $K=\bigcap U_{n}(n \in N)$. To construct our sequence $\left\{f_{n} \mid n \in N\right\}$ we take a weak cover $\mathscr{V}_{1}^{*}$ of $K$ by a finite family of weakly open subsets of $B_{1}$ such that $\left\|g(k)-g\left(k^{\prime}\right)\right\|<1$ for all $k, k^{\prime} \in V^{*} \cap K$ where $V^{*} \in \mathscr{V}_{1}^{*}$. With $\left\{U_{n} \mid n \in N\right\}$ as above, set $\mathscr{V}_{1}=\left\{U_{1} \cap V^{*} \mid V^{*} \in \mathscr{V}_{1}^{*}\right\}$. Only a finite number of linear functionals are required to define the weak open sets of $\mathscr{V}_{1}$; thus $B_{1}$ may be mapped into a finite dimensional space $E_{1}$ by a linear mapping $h$ so that each $V \in \mathscr{V}_{1}$ is the inverse image of an open set, $h(V)$, in $E_{1}$. Take a $C^{\infty}$ partition of unity subordinate to the cover $\left\{g(V) \mid V \in \mathscr{V}_{1}\right\} \cup\left(E_{1} \mid h(K)\right)$ of $E_{1}$. This $C^{\infty}$ partition of unity on $E_{1}$ induces a $C^{\infty}$ partition of unity on $B_{1}$ subordinate to $\mathscr{V}_{1}$ $\cup h^{-1}\left(E_{1} \mid h(K)\right)$. We proceed as in the construction of $f_{1}$. The construction proceeds by induction as before, the only difference being that instead of insisting that the elements of $\mathscr{V}_{n}$ be of diameter less than $2^{1-n}$, we insist that the elements of $\mathscr{V}_{n}$ be contained in $U_{n}$. This assures that condition (iii) is fulfilled since $K=\bigcap U_{n}(n \in N)$. The above prepares the way for a proof of the following.

Theorem 3.1. Let $B$ be the direct internal sum of Banach spaces $B_{1}$ and $B_{2}$. Let each of $B_{1}$ and $B_{2}$ satisfy at least one of the conditions of being separable or admitting $C^{p}$ partitions of unity subordinate to any open cover. Let $K_{1}$ be a compact subset of $B_{1}$ and let $K=\left\{k_{1}+g\left(k_{1}\right) \mid k_{1} \in K_{1}\right\}$ where $g$ is a continuous map of $K_{1}$ into $B_{2}$. Then there is a homeomorphism $d$ of $B$ onto $B$ such that

(i) $d(k)=\pi_{1}(k)$ for all $k \in K$, where $\pi_{1}$ is the natural projection of $B$ into $B_{1}$ along $B_{2}$,

(ii) $\pi_{1} \circ d=\pi_{1}$,

(iii) $d$ restricted to $B \backslash K$ is a $C^{p}$ diffeomorphism of $B \backslash K$ onto $B \backslash K_{1}$.

If both spaces allow $C^{\infty}$ norms or are separable, then $p$ in (iii) may be taken to be $\infty$.

Proof. Let $K_{1}, g, B, B_{1}$ and $B_{2}$ be as in the hypothesis of Theorem 3.1. By previous arguments we know that $g$ may be extended to a function $f$ mapping $B_{1}$ into $B_{2}$ such that $f$ is smoothly approximable by at least $C^{p}$ functions on $U=B_{1} \mid K_{1}$. Applying Lemma 2.5 we obtain the mapping $d$ which satisfies the conclusions of Theorem 3.1.

4. Smooth extensions. We may now easily prove the following result on extensions.

THEOREM 4.1. Let $B$ be $C_{0}(\omega)$ or $l_{p}(\omega)$ and let $M$ and $N$ be compact subsets of $B$. Let $f$ be a homeomorphism mapping $M$ onto $N$. There is an extension $f^{*}$ of $f$ to a homeomorphism of $B$ onto itself such that $f^{*}$ restricted to $B \backslash M$ is a $C^{\infty}$ diffeomorphism of $B \backslash M$ onto $B \backslash N$. 
Proof. First we consider a special case. Let $B$ be a separable Banach space written as the direct internal sum of subspaces $B_{1}$ and $B_{2}$. Let $M$ be a compact subset of $B_{1}$ and $N$ a compact subset of $B_{2}$. Let $g$ be a homeomorphism from $M$ to $N$. We show $g$ may be extended to a homeomorphism $g^{*}$ of $B$ onto $B$ such that $g *$ restricted to $B \backslash M$ is a $C^{\infty}$ diffeomorphism of $B \backslash M$ onto $B \backslash N$.

To construct $g^{*}$ we apply Theorem 3.1 with $B_{1}$ and $B_{2}$ as above and with $K=\{m+g(m) \mid m \in M\}$ and $K_{1}=\pi_{1}(K)=M$. The function $g$ of Theorem 3.1 is as above. By Theorem 3.1 there is a homeomorphism $d_{1}$ of $B$ onto itself satisfying

(i) $d_{1}(k)=d_{1}\left(\pi_{1}(k)\right)=g\left(\pi_{1}(k)\right)=\pi_{1}(k)$ for all $k \in K$ or, equivalently, $d_{1}(m+g(m))$ $=m$ for all $m \in M$,

(ii) $\pi_{1} \circ d_{1}=\pi_{1}$

(iii) $d_{1}$ restricted to $B \backslash K=\{m+g(m) \mid m \in M\}$ is a $C^{\infty}$ diffeomorphism of $B \backslash K$ onto $B \backslash K_{1}=B \backslash M$.

Interchanging the roles of $B_{1}$ and $B_{2}$ and thinking of $K$ as the graph of $g^{-1}$, $K=\left\{n+g^{-1}(n) \mid n \in N\right\}=\{m+g(m) \mid m \in M\}$, we apply Theorem 3.1 again to obtain a homeomorphism $d_{2}$ if $B$ onto itself satisfying

(i') $d_{2}\left(n+g^{-1}(n)\right)=n$ for all $n \in N$,

(ii') $\pi_{2} \circ d_{2}=\pi_{2}$,

(iii') $d_{2}$ restricted to $B \backslash K$ is a $C^{\infty}$ diffeomorphism of $B \backslash K$ onto $B \backslash N$.

A calculation shows that $d_{2} \circ\left(d_{1}^{-1}\right)(m)=d_{2}(m+g(m))=g(m)$ for all $m \in M$. Thus $g^{*}=d_{2} \circ\left(d_{1}^{-1}\right)$ is an extension of $g$ to a homeomorphism of $B$ onto $B$. Conditions (iii) and (iii') show that $g^{*}$ restricted to $B \backslash M$ is a $C^{\infty}$ diffeomorphism of $B \backslash M$ onto $B \backslash N$.

To complete the proof of Theorem 4.1 we reduce the general extension problem to the special case considered above by using the hypothesis that $B$ is $C_{0}(\omega)$ or $l_{p}(\omega)$. Let $f, M, N$ and $B$ be as in the hypotheses of Theorem 4.1. By the Splitting Theorem we write $B$ as the direct sum of isomorphic subspaces $B_{1}$ and $B_{2}$ so that $\pi_{1}$ determines a homeomorphism of $K=M \cup N$ onto $K_{1}=\pi_{1}(K)$. Then there is a continuous function $g$ mapping $K_{1}$ into $B_{2}$ such that $K=\left\{k_{1}+g\left(k_{1}\right) \mid k_{1} \in K_{1}\right\}$. Since $B$ is separable so are $B_{1}$ and $B_{2}$. Theorem 3.1 in its alternative reading applies. Let $d$ be a homeomorphism of $B$ onto itself satisfying the conclusions of the alternative reading of Theorem 3.1 .

Since $B_{1}$ and $B_{2}$ are isomorphic we can and will let $h$ denote a linear isometry of $B_{1}$ onto $B_{2}$. The result established above now applies to the homeomorphism $g=h \circ d \circ f \circ\left(d^{-1}\right)$ of $d(M)$ onto $h(d(N))$. Thus we may extend $g$ to $g^{*}$ as above. Calculation shows that $f^{*}=\left(d^{-1}\right) \circ\left(h^{-1}\right) \circ g^{*} \circ d$ is an extension of $f$ which has the properties required in the conclusion of Theorem 4.1. Thus Theorem 4.1 is proved.

\section{REFERENCES}

1. R. D. Anderson, On a theorem of Klee, Proc. Amer. Math. Soc. 17 (1966), 1401-1404. MR 34 \#4864. 
2. R. D. Anderson, Strongly negligible sets in Fréchet manifolds, Bull. Amer. Math. Soc. 75 (1969), 64-67. MR 38 \#6634.

3. C. Bessaga, Every infinite dimensional Hilbert space is diffeomorphic with its unit sphere, Bull. Acad. Polon. Sci. Sér. Sci. Math. Astronom. Phys. 14 (1966), 27-31. MR 33 \#1862.

4. -_ , Topics from infinite dimensional topology, Lecture Notes Series No. 18, Matematisk Institut, Aarhus, 1969.

5. Robert Bonic and John Frampton, Smooth functions on Banach manifolds, J. Math. Mech. 15 (1966), 877-898. MR 33 \#6647.

6. H. H. Corson, Thin sets in Fréchet spaces, Sympos. on Infinite Dimensional Topology (Baton Rouge, 1967), Ann. of Math. Studies, Princeton Univ. Press, Princeton, N. J., 1971.

7. W. H. Cutler, Negligible subsets of infinite-dimensional Fréchet manifolds, Proc. Amer. Math. Soc. 23 (1969), 668-675. MR 40 \#2133.

8. Jean Dieudonné, Foundations of modern analysis, Pure and Appl. Math., vol. 10, Academic Press, New York, 1960. MR 22 \#11074.

9. I. Edelstein, B. Mitjagin and E. Semenov, The linear groups of $C$ and $L_{1}$ are contractible, Bull. Acad. Polon. Sci. Sér. Sci. Math. Astronom. Phys. 18 (1969), 27-33.

10. J. Eells and K. D. Elworthy, Open embeddings of certain Banach manifolds, Ann. of Math. (2) 91 (1970), 465-485. MR 41 \#7725.

11. J. Eells, Jr. and N. Kuiper, Homotopy negligible subsets, Compositio Math. 21 (1969), 155-161. MR 40 \#6546.

12. Victor L. Klee, Jr., Convex bodies and periodic homeomorphisms in Hilbert space, Trans. Amer. Math. Soc. 74 (1953), 10-43. MR 14, 989.

13. N. H. Kuiper, The homotopy type of the unitary group of a Hilbert space, Topology 3 (1965), 19-30. MR 31 \#4034.

14. Nicolaas Kuiper and Dan Burghelea, Hilbert manifolds, Ann. of Math. (2) 90 (1969), 379-417. MR 40 \#6589.

15. Serge Lang, Introduction to differentiable manifolds, Interscience, New York, 1962. MR 27 \#5192.

16. P. A. Martens, Representations of infinite dimensional manifolds and $\infty-p$ homology functors, Bull. Amer. Math. Soc. 76 (1970), 641-645. MR 41 \#6247.

17. N. Moulis, Sur les variétés Hilbertiennes et les fonctions non dégénérés, Nederl. Akad. Wetensch. Proc. Ser. A 71 = Indag. Math. 30 (1968), 497-511. MR 40 \#8083.

18. G. Neubauer, Der Homotopietyp der Automorphismengruppe in den Räumen $l_{p}$ und $c_{0}$, Math. Ann. 174 (1967), 33-40. MR 36 \#2171.

19. P. Renz, Smooth extensions and extractions in Banach spaces, Notices Amer. Math. Soc. 16 (1969), 557. Abstract \#666-21.

20. - Smooth extensions and smooth extractions, Thesis, University of Washington, Seattle, Wash., 1969.

Department of Mathematics, Wellesley College, Wellesley, Massachusetts 02181 